\title{
Coagulopathy in COVID-19: Connecting the Dots Together
}

\author{
Ajay Gandhi ${ }^{1}$ Klaus Görlinger ${ }^{2,3}$ \\ ${ }^{1}$ Clinical Affairs, Instrumentation Laboratory India Private Limited, \\ New Delhi, India \\ ${ }^{2}$ Department of Anesthesiology and Intensive Care Medicine, \\ University Hospital Essen, Essen, Germany \\ ${ }^{3}$ Medical Director, Tem Innovations GmbH, Munich, Germany
}

\begin{abstract}
Address for correspondence Ajay Gandhi, Clinical Affairs, MD (Pathology), Instrumentation Laboratory India Private Limited, 1471-76, Agrawal Millennium Tower II, Plot Number E-4, Netaji Subhash Place, Pitampura, New Delhi 110034, India (e-mail: agandhi@werfen.com).
\end{abstract}

J Card Crit Care:2020;4:47-50

\author{
Abstract \\ Keywords \\ - coagulopathy \\ - COVID-19 \\ - COVID-19-associated \\ coagulopathy
}

Coronavirus disease 2019 (COVID-19) caused by SARS-CoV-2 (severe acute respiratory syndrome coronavirus 2) virus has spread quickly and become a public health emergency of global concern. Originating in the Wuhan district of China, which has reportedly been declared free of it now, the rest of the world continues to struggle with its severity and spread. While a lot of scientific publications and clinical data are available, newer clinical investigations and experiences continue to evolve, thereby depicting the dynamic nature of the disease and the knowledge around it. Researchers and clinical professionals continue to collect scientific information, clinical data, and evidence to help build a knowledge pool and guidance for the health care professionals to manage those affected with this pandemic disease. As significant and new data emerge, a lot of already available information gets confirmed and updated, while some of it also getting rejected or disapproved. In this article, we aim to put together the scientific and clinical information that is proven so far and the areas where more data or evidence is needed before a clear understanding can be achieved and guidance can be developed.

\section{Background}

Of the several viral epidemics that occurred in the past, such as the severe acute respiratory syndrome coronavirus (SARS-CoV) in 2002, H1N1 influenza in 2009, and Middle East respiratory syndrome coronavirus (MERS-CoV) in Saudi Arabia in 2012, the SARS-CoV-2 virus and the associated Coronavirus disease 2019 (COVID-19) disease has been the most contagious one, with a rapid spread across the globe. ${ }^{1}$ As far as the available data suggests, most of the infected patients either have subclinical or mild clinical symptoms, and a small patient population suffers from severe disease manifestations, with viral sepsis leading to single or multiple organ failure or even death being the most common issue. ${ }^{2}$ COVID-19-associated coagulopathy (CAC), as has become a prevalent term now, is one of the most common hemostaseology findings and is associated with adverse outcomes. ${ }^{3}$ Accordingly, coagulopathy is one of the most significant prognostic factors in COVID-19. Risk stratification and triage based on predictive laboratory parameters such as D-dimer, IL-6, lymphocyte count, and LDH, as well as clinical scoring systems such as the Sequential Organ Failure Assessment (SOFA) score, Sepsis-Induced Coagulopathy (SIC) score, and International Society of Thrombosis and Haemostasis Disseminated Intravascular Coagulation (ISTH DIC) score, can help health care professionals and institutions in managing COVID-19 patients and hospital resources such as intensive care unit (ICU) beds, intubation, and ventilator therapy, as well as extracorporeal membrane oxygenation (ECMO) in the best way, particularly in times of exponential growth of infected people and limited hospital resources.

\section{Pathogenesis}

SARS-CoV-2 virus primarily affects epithelial cells in the respiratory tract as well as vascular endothelial cells in case of viremia. The virus enters the cells through the angiotensin-converting enzyme 2 (ACE2) receptors, which are most
DOI https://doi.org/

10.1055/s-0040-1712739

ISSN 2457-0206.
License terms

()(1) $\Theta \circledast$ 
commonly found in the alveolar epithelial cells followed by endothelial cells. ${ }^{4}$ Other organs presenting these receptors are the renal and the gastrointestinal tracts. Furthermore, transmembrane protease serine subtype 2 (TMPRSS2) is a critical factor enabling cellular infection by coronaviruses including SARS-CoV-2 and is also the most frequently altered gene in primary prostate cancer. The modulation of its expression by sex steroids could contribute to the male predominance of severe COVID-19., ${ }^{5,6}$ At the same time, this is the rationale for serine protease inhibitors such as camostat as a potential therapeutic intervention (NCT04321096 and NCT04338906).

The incubation period for COVID-19 is around 5 to 14 days. ${ }^{7}$ The viral infection is capable of triggering an excessive immune reaction in the host, which can, in some patients, result in a "cytokine storm" presenting clinically as hyperinflammation. ${ }^{8}$ The effect is extensive tissue damage and vascular leakage. One protagonist of this "cytokine storm" is interleukin-6 (IL-6). IL-6 is produced by activated leukocytes and endothelial cells and acts on multiple cells and tissues. ${ }^{9,10}$ Furthermore, IL-6 is also involved in the pathogenesis of the cytokine release syndrome (CRS), which is an acute systemic inflammatory syndrome characterized by fever and multiple organ dysfunction.

The pathological and clinical changes manifested in patients with COVID-19 can be ascribed mostly to the innate immune response. Here, an imbalance between the non-specific innate immune response (hyperinflammation and activation of macrophages and neutrophil granulocytes) and the specific adaptive immune response (production of specific antibodies by lymphocytes) seems to play an important role. ${ }^{11,12}$ Accordingly, high IL-6 levels and low lymphocyte counts are associated with poor outcome in COVID-19. ${ }^{13,14}$ This immunological imbalance is typical for older patients (immunosenescence) and patients with chronic inflammatory diseases (inflammaging). ${ }^{15-18}$

\section{Clinicopathological Manifestations}

\section{Hypercoagulability}

There is a growing body of evidence that hypercoagulability is one of the most common pathological manifestation of COVID-19.' However, the real incidence of macro- or microthrombosis is not known yet. A potential mechanism is the upregulation of tissue factor expression on circulating monocytes, thrombopoietin, and fibrinogen, as well as downregulation of plasminogen activator inhibitor type 1 (PAI-1) by IL-6. ${ }^{19}$ This results in increased and delocalized thrombin generation as well as increased clot firmness and stability (fibrinolysis resistance). Accordingly, increased D-dimer levels $(>1 \mu \mathrm{g} / \mathrm{mL}$ ) are a good predictor for poor outcome in COVID-19..$^{20,21}$ Due to hemoconcentration, vascular endothelial cell injury, and hypercoagulable state of patients with COVID-19-particularly in patients with obesity, advanced age, and other risk factors-risk of thrombosis is increased in this patient population. Therefore, the risk of venous thromboembolism cannot be ignored during the course and treatment of COVID-19.22 Thromboprophylaxis is recommended in all hospitalized COVID-19 patients. ${ }^{23}$ Tang et al reported that patients with SIC score $\geq 4$ or D-dimer $>3 \mu \mathrm{g}$ / $\mathrm{mL}$ (sixfold of the upper limit of normal) showed a significant reduction in 28 -day mortality ( 40 vs. $64.2 \%, p=0.029$; and 32.8 vs. $52.4 \%, p=0.017$, respectively). ${ }^{24}$ No difference in 28-day mortality was found between heparin users and nonusers in the overall population of severe COVID-19 patients (30.3 vs. $29.7 \%$; $p=0.910$ ). Intensified anticoagulation was performed by administering low molecular weight heparin (40-60 mg enoxaparin/day) or unfractionated heparin (10,000-15,000 U/day) for at least 7 days. As a matter of fact, data is not available for direct oral anticoagulants in COVID-19 patients.

\section{Diffuse Microvascular Damage}

Multiple organ failure caused by diffuse microvascular damage and microthrombosis is an important cause of death in critically ill patients with COVID-19 and may be related to CRS and immune imbalance. ${ }^{25}$ Around $70 \%$ of COVID-19 nonsurvivors and $0.6 \%$ of survivors meet the ISTH DIC diagnostic criteria during their hospital stay. ${ }^{26}$ Most of them present a hypercoagulable state. However, it is unclear whether the pathophysiology of SIC, DIC, and CAC is the same and whether the terms can be used interchangeably. ${ }^{27}$

\section{Bleeding Risk}

Notably, some COVID-19 patients may also have an increased bleeding risk due to imbalances in platelet production and consumption and other coagulation disorders. ${ }^{28,29}$ However, bleeding complications have been reported rarely in COVID-19 patients. It is under investigation whether bleeding complications might occur in a specific subset of COVID-19 patients (medication, comorbidities) or at a specific stage of the disease (severe, late stage).

\section{Laboratory Investigations for Coagulopathy in COVID-19}

\section{D-Dimer}

Elevated D-dimers can occur in $50 \%$ of patients with COVID-19, and fibrinogen degradation products and D-dimers are significantly higher in severe patients and nonsurvivors compared with mild patients and survivors..$^{30}$ Accordingly, elevated D-dimers have to be considered as a marker of poor outcome in patients with COVID-19 infection. ${ }^{20,25}$ In patients with markedly increased D-dimers (which may be arbitrarily defined as three- to fourfold of the upper limit of normal), admission to hospital should be considered even in the absence of other severity symptoms since it has to considered as a marker of poor outcome. ${ }^{31}$

\section{Platelet Count}

Thrombocytopenia is a prominent marker of severity or mortality associated with sepsis. However, most patients with COVID-19 have platelet count in the normal range, ${ }^{7}$ although the incidence of thrombocytopenia varies, the numbers falling with increasing severity. ${ }^{32}$ 


\section{Prothrombin Time}

A slight prolongation of prothrombin time (PT) has been observed in severe stages of COVID-19 or in nonsurvivors at the time of admission. ${ }^{1,2,31,33}$ This is one of the commonly available laboratory coagulation parameters that could serve as predictor of ICU admission.

\section{Fibrinogen}

As an acute response protein, fibrinogen may be increased in the course of mild disease and in the early stages of severe patients and can be significantly reduced in the late stages of severe patients. However, the increase in fibrinogen levels is usually less pronounced in viral compared with bacterial sepsis. Nonetheless, fibrinogen forms another significant marker while monitoring DIC or CAC. ${ }^{33}$

Hence, for specific monitoring of evolving or established coagulopathy, coagulation laboratory parameters such as D-dimer, platelet count, PT, and fibrinogen are required not only at the time of hospital admission but also during hospital stay for all patients with suspected or confirmed COVID-19. ${ }^{34}$

Actually, data on the utility of viscoelastic testing devices such as rotational thromboelastometry (ROTEM) and thromboelastography (TEG) in CAC are limited but promising. The value of ROTEM in predicting the clinical course, need for hospital resources (ICU beds, respiratory therapy, ECMO, etc.), and outcomes in hospitalized patients with COVID19 will be assessed in the ongoing ROHOCO study. Further research is needed to investigate whether ROTEM/TEG is useful in identifying COVID-19 patients who might benefit from therapeutic anticoagulation and to guide hemostatic therapy in patients with hyper- and hypocoagulability. ${ }^{35}$

\section{Conflict of Interest}

None.

\section{References}

1 Huang C, Wang Y, Li X, et al. Clinical features of patients infected with 2019 novel coronavirus in Wuhan, China. Lancet 2020;395(10223):497-506

2 Chen N, Zhou M, Dong X, et al. Epidemiological and clinical characteristics of 99 cases of 2019 novel coronavirus pneumonia in Wuhan, China: a descriptive study. Lancet 2020;395(10223):507-513

3 Simmons J, Pittet JF. The coagulopathy of acute sepsis. Curr Opin Anaesthesiol 2015;28(2):227-236

4 Letko M, Marzi A, Munster V. Functional assessment of cell entry and receptor usage for SARS-CoV-2 and other lineage B betacoronaviruses. Nat Microbiol 2020;5(4):562-569

5 Stopsack KH, Mucci LA, Antonarakis ES, Nelson PS, Kantoff PW. TMPRSS2 and COVID-19: serendipity or opportunity for intervention? Cancer Discov 2020 (e-pub ahead of print). doi:10.1158/2159-8290.CD-20-0451

6 Hoffmann M, Kleine-Weber H, Schroeder S, et al. SARS-CoV-2 cell entry depends on ACE2 and TMPRSS2 and is blocked by a clinically proven protease inhibitor. Cell 2020;181(2): 271-280.e8

7 Guan WJ, Ni ZY, Hu Y, et al; China Medical Treatment Expert Group for Covid-19. Clinical characteristics of coronavirus disease 2019 in China. N Engl J Med 2020 (e-pub ahead of print). doi: 10.1056/NEJMoa2002032
8 Ruan Q, Yang K, Wang W, Jiang L, Song J. Clinical predictors of mortality due to COVID-19 based on an analysis of data of 150 patients from Wuhan, China. Intensive Care Med 2020 (e-pub ahead of print). doi: 10.1007/s00134-020-05991-x

9 Almeida GD, Porada CD, St Jeor S, Ascensao JL. Human cytomegalovirus alters interleukin- 6 production by endothelial cells. Blood 1994;83(2):370-376

10 Liu Q Imaizumi T, Aizawa T, et al. Cytosolic sensors of viral RNA are involved in the production of interleukin- 6 via tolllike receptor 3 signaling in human glomerular endothelial cells. Kidney Blood Press Res 2019;44(1):62-71

11 Mogensen TH. Pathogen recognition and inflammatory signaling in innate immune defenses. Clin Microbiol Rev 2009;22(2):240-273

12 Liu T, Zhang L, Joo D, et al. NF-kB signaling in inflammation. Signal Transduct Target Ther 2017;2:17023

13 Wang D, Hu B, Hu C, et al. Clinical characteristics of 138 hospitalized patients with 2019 novel coronavirusinfected pneumonia in Wuhan, China. JAMA 2020 (e-pub ahead of print). doi: 10.1001/jama.2020.1585

14 Henry BM. COVID-19, ECMO, and lymphopenia: a word of caution. Lancet Respir Med 2020;8(4):e24

15 Ventura MT, Casciaro M, Gangemi S, Buquicchio R. Immunosenescence in aging: between immune cells depletion and cytokines up-regulation. Clin Mol Allergy 2017;15-21

16 Frasca D, Blomberg BB. Inflammaging decreases adaptive and innate immune responses in mice and humans. Biogerontology 2016;17(1):7-19

17 Wang Y, Wang Y, Chen Y, Qin Q. Unique epidemiological and clinical features of the emerging 2019 novel coronavirus pneumonia (COVID-19) implicate special control measures. J Med Virol 2020 (e-pub ahead of print). doi: 10.1002/jmv.25748

18 Lin L, Lu L, Cao W, Li T. Hypothesis for potential pathogenesis of SARS-CoV-2 infection-a review of immune changes in patients with viral pneumonia. Emerg Microbes Infect 2020;9(1):727-732

19 Mei H, Hu Y. Characteristics, causes, diagnosis and treatment of coagulation dysfunction in patients with COVID-19 [in Chinese]. Zhonghua Xue Ye Xue Za Zhi 2020;41(0):E002

20 Zhou F, Yu T, Du R, et al. Clinical course and risk factors for mortality of adult inpatients with COVID-19 in Wuhan, China: a retrospective cohort study. Lancet 2020;395(10229): 1054-1062

21 Han H, Yang L, Liu R, et al. Prominent changes in blood coagulation of patients with SARS-CoV-2 infection. Clin Chem Lab Med 2020 (e-pub ahead of print). doi:10.1515/cclm-2020-0188

22 Klok FA, Kruip MJHA, van der Meer NJM, et al. Incidence of thrombotic complications in critically ill ICU patients with COVID-19. Thromb Res 2020 (e-pub ahead of print). doi: $10.1016 /$ j.thromres.2020.04.013

23 Hunt B, Retter A, McClintock C. Practical guidance for the prevention of thrombosis and management of coagulopathy and disseminated intravascular coagulation of patients infected with COVID-19. https://thrombosisuk.org/downloads/T\&H\%20and\%20COVID.pdf. Accessed April 20, 2020

24 Tang N, Bai H, Chen X, Gong J, Li D, Sun Z. Anticoagulant treatment is associated with decreased mortality in severe coronavirus disease 2019 patients with coagulopathy. J Thromb Haemost 2020 (e-pub ahead of print). doi: 10.1111/jth.14817

25 Tang N, Li D, Wang X, Sun Z. Abnormal coagulation parameters are associated with poor prognosis in patients with novel coronavirus pneumonia. J Thromb Haemost 2020;18(4):844-847

26 Jiang H, Liu L, Guo T, et al. Improving the safety of CAR-T cell therapy by controlling CRS-related coagulopathy. Ann Hematol 2019;98(7):1721-1732

27 Iba T, Levy JH, Warkentin TE, Thachil J, van der Poll T, Levi M; Scientific and Standardization Committee on DIC, and the Scientific and Standardization Committee on Perioperative 
and Critical Care of the International Society on Thrombosis and Haemostasis. Diagnosis and management of sepsisinduced coagulopathy and disseminated intravascular coagulation. J Thromb Haemost 2019;17(11):1989-1994

28 Magdi M, Rahil A. Severe immune thrombocytopenia complicated by intracerebral haemorrhage associated with coronavirus infection: a case report and literature review. Eur J Case Rep Intern Med 2019;6(7):001155

29 CascellaM, RajnikM, CuomoA, et al. Features, Evaluation and Treatment Coronavirus (COVID-19). Treasure Island, FL: StatPearls Publishing; 2020

30 Gao Y, Li T, Han M, et al. Diagnostic utility of clinical laboratory data determinations for patients with the severe COVID-19. J Med Virol 2020;(e-pub ahead of print). doi: 10.1002/jmv.25770

31 Thachil J, Tang N, Gando S, et al. ISTH interim guidance on recognition and management of coagulopathy in COVID-19. J Throm Haemost; 2020 (e-pub ahead of print). doi:10.1111/ JTH.14810
32 Chen G, Wu D, Guo W, et al. Clinical and immunologic features in severe and moderate forms of coronavirus disease 2019. MedRxiv 2020 (e-pub ahead of print). doi:10.1101/2020.02.16.20023903

33 Wada H, Thachil J, Di Nisio M, et al Scientific Standardization Committee on DIC of the International Society on Thrombosis Haemostasis. Guidance for diagnosis and treatment of DIC from harmonization of the recommendations from three guidelines. J Thromb Haemost 2013;11:761-767

34 Lippi G, Plebani M. Laboratory abnormalities in patients with COVID-2019 infection. Clin Chem Lab Med 2020 (e-pub ahead of print). doi: 10.1515/cclm-2020-0198

35 Song JC, Wang G, Zhang W, et al. People's Liberation Army Professional Committee of Critical Care Medicine, Chinese Society on Thrombosis and Haemostasis. Chinese expert consensus on diagnosis and treatment of coagulation dysfunction in COVID-19. Mil Med Res 2020;7:19 